\title{
Hepatic Arterial Infusion for Unresectable Liver Metastases from Colorectal Cancer: The Dawn of a New Era?
}

\author{
Paul J. Karanicolas, MD, PhD, FRCSC, FACS ${ }^{1,2}$ and Yoo-Joung Ko, MD, MMSc, SM, FRCPC ${ }^{3,4}$ \\ ${ }^{1}$ Division of Surgical Oncology, Odette Cancer Centre, Sunnybrook Health Sciences Centre, Toronto, Canada; \\ ${ }^{2}$ Department of Surgery, University of Toronto, Toronto, Canada; ${ }^{3}$ Division of Medical Oncology, Odette Cancer Centre, \\ Sunnybrook Health Sciences Centre, Toronto, Canada; ${ }^{4}$ Division of Medical Oncology, University of Toronto, Toronto, \\ Canada
}

Nearly 10 years ago, authors of a meta-analysis examining the effectiveness of hepatic arterial infusion (HAI) chemotherapy for patients with unresectable colorectal liver metastases (CRLM) concluded that it was "the end of an era." ${ }^{1}$ Although patients treated with HAI achieved an impressive improvement in their tumor response rate (43 vs. $18 \%$ ), no benefit was seen in the ultimate patientimportant outcome of overall survival (OS). Despite the provocative title, the role of HAI for patients with unresectable CRLM continued to be explored at a handful of high-volume, specialized centers. Recent reports of excellent long-term outcomes from patients treated at the Memorial Sloan-Kettering Cancer Center (MSKCC) and the University of Pittsburgh Medical Center (UPMC) suggest that HAI may indeed provide important benefit for selected patients with unresectable CRLM, even in an era of better systemic therapy.,

In the accompanying article, Zureikat and colleagues from UPMC report long-term outcomes for 40 patients treated in the contemporary era of systemic therapy (2004$2014)$ with a combination of HAI and systemic therapy. ${ }^{2}$ The comparator group, consisting of 46 patients with liveronly metastases who received systemic therapy alone, is problematic for three reasons. First, the outcomes are surprisingly poor. The median survival time was 15.3 months from diagnosis compared with an OS in the range of 24-28 months for patients treated in other trials with modern

(C) Society of Surgical Oncology 2016

First Received: 3 July 2016;

Published Online: 14 July 2016

P. J. Karanicolas, MD, PhD, FRCSC, FACS

e-mail: paul.karanicolas@sunnybrook.ca systemic therapy regimens. ${ }^{4,5}$ The authors hypothesize that the poor OS for these patients may be attributable to a heavy burden of liver metastases. However, it should be noted that none of these patients had documented extrahepatic metastases, so if anything, their outcomes should have been at least as good as those for patients with diffuse colorectal metastases. Second, the patients in the comparator group received treatment at more than 21 network community sites affiliated with UPMC, whereas the patients in the HAI group received treatment at the UPMC flagship oncologic facility. Finally, the primary tumor was resected in all the patients treated with HAI, whereas only $54 \%$ of the patients in the comparator group underwent resection. These differences in baseline characteristics and concurrent therapies make it difficult to compare the outcomes between the treatment groups. Indeed, the survival curves appear to separate early on, less than 6 months after diagnosis, at which point most patients in the HAI group would not even have undergone an operation to implant the pump.

Despite these limitations, the outcomes for the 40 patients treated with HAI offer a glimpse into what is possible with this intensive therapy in a modern chemotherapy setting. The median OS for the patients treated with HAI plus systemic therapy was 32.8 months, substantially better than the outcomes expected with any modern systemic therapy regimen. Interestingly, only three patients achieved a response sufficient to allow resection, perhaps due to the high burden of baseline hepatic disease (median 15 tumors, $40 \%$ replacement of liver parenchyma). The extent of downsizing also may have been influenced by the use of HAI in the second-line setting in this series. The patients received a median of only four HAI therapy cycles, with a maximum of seven cycles. 
Downsizing to resectability may have been more successful if HAI had been used in untreated patients or those with a lower burden of disease.

The results of this series compare favorably with those reported in the recent publication from MSKCC and provide some reassurances that HAI can be safely and effectively delivered outside this single, highly experienced institution. ${ }^{3}$ Although the patient population and interventions are similar, the two studies have some notable differences. D'Angelica and colleagues at MSKCC performed a prospective, phase 2 clinical trial for HAI combined with systemic therapy, using conversion to resectability as the primary outcome. The patient characteristics and burden of liver metastases were similar in the two studies, although the MSKCC group included a substantial proportion of patients $(35 \%)$ who had not received prior chemotherapy. Perhaps as a result of this, the patients in the MSKCC cohort received more cycles of HAI and were more likely to undergo resection (47\% resected at a median of 6 months from treatment initiation). The OS was longer in the entire MSKCC cohort (38 months), but very similar in the group of patients who did not undergo resection (32 months).

Interpreting these two studies concurrently, it is clear that HAI is capable of tremendous tumor response in the liver and, in selected patients, can achieve excellent longterm survival. The remaining challenges are to differentiate the effect of HAI from careful patient selection and to identify the patients who are most likely to benefit from this therapy. Ultimately, this can be accomplished only with a well-designed, prospective, randomized controlled trial. Ten such trials have already been performed but were summarily dismissed because they did not combine HAI with modern systemic therapy and failed to show a significant improvement in OS. Several more centers across North America and Europe have now started offering HAI in combination with systemic therapy, and a multicenter trial seems feasible, if not imminent. The era of HAI monotherapy has indeed ended. Are we witnessing the dawn of the HAI plus systemic therapy era? Only time will tell.

\section{REFERENCES}

1. Mocellin S, Pilati P, Lise M, Nitti D. Meta-analysis of hepatic arterial infusion for unresectable liver metastases from colorectal cancer: the end of an era? J Clin Oncol. 2007;25:5649-54.

2. Dhir M, Jones H, Shuai $\mathrm{Y}$, et al. Hepatic arterial infusion in combination with modern systemic chemotherapy is associated with improved survival compared to modern systemic chemotherapy alone in patients with isolated unresectable colorectal liver metastases: a case control study. Ann Surg Oncol (in press).

3. D'Angelica MI, Correa-Gallego C, Paty PB, et al. Phase II trial of hepatic artery infusional and systemic chemotherapy for patients with unresectable hepatic metastases from colorectal cancer: conversion to resection and long-term outcomes. Ann Surg. 2015;261:353-60.

4. Heinemann V, von Weikersthal LF, Decker T, et al. FOLFIRI plus cetuximab versus FOLFIRI plus bevacizumab as first-line treatment for patients with metastatic colorectal cancer (FIRE-3): a randomised, open-label, phase 3 trial. Lancet Oncol. 2014;15: $1065-75$

5. Cremolini C, Loupakis F, Antoniotti C, et al. FOLFOXIRI plus bevacizumab versus FOLFIRI plus bevacizumab as first-line treatment of patients with metastatic colorectal cancer: updated overall survival and molecular subgroup analyses of the openlabel, phase 3 TRIBE study. Lancet Oncol. 2015;16:1306-15. 